\title{
Effect of the rotor shape on the mixing characteristics of a continuous flow Taylor- vortex reactor
}

\author{
Oliver Richter, Heike Hoffmann, Bettina Kraushaar-Czarnetzki* \\ Institute of Chemical Process Engineering CVT, University of Karlsruhe, Kaiserstrasse 12, D-76128 Karlsruhe, Germany
}

\section{A R T I C L E I N F O}

\section{Article history:}

Received 23 October 2007

Received in revised form 28 March 2008

Accepted 3 April 2008

Available online 6 April 2008

\section{Keywords:}

Taylor-Couette reactor

Dispersion

Mass transfer

Mixing

Rotor shape

\begin{abstract}
A B S T R A C T
Macro- and micromixing in a continuous flow Taylor-vortex reactor with novel ribbed rotors were investigated and compared to the features of a classical cylindrical rotor. The characterisation was performed in a wide hydrodynamic range $(40<T a<2500$ and $0.03<R e<0.51)$ through tracer experiments and the analysis of the rotor power consumption. Additionally, the flow patterns were visualised by using a rheoscopic fluid. The results show that the novel rotors equipped with ribs immobilise and stabilise the vortices. As compared to cylindrical rotors, micromixing is clearly enhanced while axial dispersion can be simultaneously reduced. Through the use of ribbed rotors, the operational window can be broadened considerably, in which the reactor runs at very low or moderate extent of macromixing.
\end{abstract}

(c) 2008 Elsevier Ltd. All rights reserved.

\section{Introduction}

A Taylor-vortex reactor consists of two concentrical cylinders (Fig. 1, left). In most devices, the outer cylinder is static (stator) and the inner rotates (rotor).

Different flow patterns can be formed in the annular gap depending on the rotation speed and the resulting centrifugal forces (Andereck et al., 1986). The rotating flow is described by the dimensionless Taylor number Ta.

$\mathrm{Ta}=\frac{r_{i} \cdot \omega \cdot\left(r_{0}-r_{i}\right)}{v}$

A laminar shear-flow, the so-called Couette flow, CF, is observed at low $\mathrm{Ta}$ numbers. The formation of counter-rotating toroidal laminar vortices with a diameter $d \approx r_{0}-r_{i}$ starts when a critical $\mathrm{Ta}$ number, $\mathrm{Ta} \mathrm{C}_{c}$, is exceeded. This regime is denoted as laminar Taylor-vortex flow, LTVF. When the empirical criterion $r_{0} / r_{i}>1.4$ is fulfilled, a further increase in Ta will cause azimuthal wavy motion, denoted as wavy vortex flow, WVF (Coles, 1965). Even higher Ta numbers promote the gradual transformation of the vortices into chaotic structures (turbulent vortex flow, TVF) and, finally, a fully developed turbulent flow.
In continuous operation mode, a fluid is passing through the annular gap of the Taylor-vortex reactor in axial direction. The axial flow is characterised with the dimensionless Reynolds number Re.

$\operatorname{Re}=\frac{u_{\mathrm{ax}} \cdot\left(r_{0}-r_{i}\right)}{v}$

The axial flux stabilises the flow regimes in that the flow transitions occur at higher Ta numbers. The toroidal vortices move downstream in stack at moderate $R e$ numbers while they are helically deformed at high $R e$ (Lueptow et al., 1992). According to theoretical considerations by Recktenwald et al. (1993) the vortex axial displacement $u_{d}$ in the LTVF regime should be marginally higher than the superficial velocity $u_{\mathrm{ax}}$, amounting to $u_{d} / u_{\mathrm{ax}}=1.14$. Induced by a bypass flow, however, the axial displacement velocity may be slowed down until total stagnancy (Giordano et al., 1998).

The advantages of a Taylor-vortex reactor, compared to classical reactor configurations, are basically the toroidal vortex flows and in consequence to construction the large specific reactor surface. Numerous applications using these benefits were reported in literature, like emulsion polymerisation (Kataoka et al., 1995; Wei et al., 2000), catalytic (Cohen and Maron, 1990), photochemical (Haim and Pismen, 1994; Forney and Pierson, 2003), electrochemical (Coeuret and Legrand, 1981) and enzymatic reactions (Iosilevskii et al., 1993; Giordano et al., 2000b), cell cultivation (Haut et al., 2003), precipitation (Jung et al., 2000; Judat et al., 2004), flocculation for wastewater treatment (Grohmann et al., 1981), dynamic tangential and membrane filtration (Schwille et al., 2002; Lee and Lueptow, 2004), microparticle classification (Ohmura et al., 2005) and liquid-liquid extraction (Baier et al., 2000; Forney et al., 2002).
* Corresponding author. Tel.: +49721 608 3947; fax: +497216086118.
E-mail address: Kraushaar@cvt.uka.de (B. Kraushaar-Czarnetzki). 

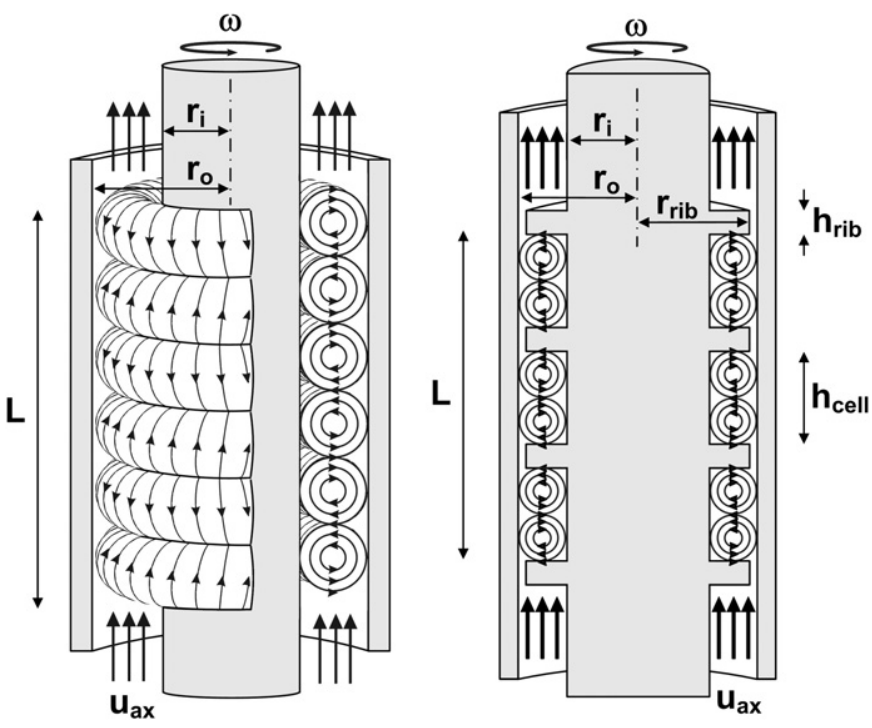

Fig. 1. Schemes of Taylor-vortex reactors used in this work; left: cylindrical rotor; right: ribbed rotor.

Successful reactor design and operation require the knowledge of the macro- and micromixing patterns, which, in case of a Taylorvortex device, are equivalent to inter- and intra-vortex mass transport. Several studies have been carried out on Taylor-vortex devices in batch mode, i.e. without axial flow, using tracer techniques (Tam and Swinney, 1987; Desmet et al., 1996a, b, 1997; Ohmura et al., 1997; Campero and Vigil, 1997), laser-optical methods (Racina et al., 2005) or chemical test reactions (Liu and Lee, 1999). In contrast to other mass transport models developed, the multi-zone model by Desmet et al. (1997) takes into account that the vortices are not always perfectly mixed. Rather, the fluid in the outer boundary (active mixing region, AMR) is dispersed more rapidly than in the core of a vortex (isolated mixing region, IMR).

Mixing in Taylor-vortex devices operated in continuous (axial) flow mode has also been the subject of numerous studies (Kataoka et al., 1975; Kataoka and Takigawa, 1981; Enokida et al., 1989; Pudjiono et al., 1992; Moore and Cooney, 1995; Giordano et al., 1998; Yim et al., 1998; Haut et al., 2003; Judat et al., 2004). In continuous flow mode, not only vortex segregation but also bypass effects and vortex slowdown can be observed. The mass transport models most suitable to take these phenomena into account are derivatives of Desmet's multi-zone model (Haut et al., 2003; Giordano et al., 2000a; Syed and Früh, 2003).

Reviewing the literature, mixing in the different vortex flow regimes at moderate Re number can be characterised as follows. In the LTVF regime, both inter-vortex (macromixing) and intra-vortex mixing (micromixing) are of low intensity. Upon transition to WVF and TVF, the extent of mixing increases drastically on all scales. Hence, with increasing $\mathrm{Ta}$ number, the system is transformed from a reactor with nearly plug-flow behaviour with a possible bypass into a well-mixed vessel.

The kinetics of many chemical reactions are such that macroscopic backmixing should be minimised in order to reduce the spacetime required for an aspired conversion level. On the other hand, intense micromixing is desirable for all types of reactions and, in particular, for multiphase systems. In a classical Taylor-vortex reactor, the intensification of micromixing cannot be decoupled from a detrimental increase in axial dispersion.

Substituting rotors with different shape for the conventional cylinder can change the mixing characteristics in Taylor-vortex devices. Rotz and Suh (1976, 1979), for instance, used rotors with a v-grooved surface to enhance laminar mixing in highly viscous fluids. Homogenising and maximising shear rates were achieved by furnishing the rotor with a lobed cross-section (Soos et al., 2007). Shape modifications investigated include concave (Wiener et al., 1997) or undulating geometries (Drozdov, 2002) and conical rotors. The latter were applied to cope with the increasing viscosity during polymerisation reactions (Moritz et al., 1999) and to enhance backmixing at low Ta numbers (Ohmura et al., 2004). Hence, in these studies, a reduction of the axial dispersion was neither intended nor observed.

The aim of the present work, however, is to find a rotor shape through which extensive backmixing on the macroscale can be suppressed even at those high $\mathrm{Ta}$ numbers required for intense micromixing. It will be shown that rotors segmented by ribs as depicted in Fig. 1 (right) are suitable to approach this goal.

\section{Experimental}

\subsection{Experimental set-up}

All experiments were run at ambient temperature $\left(24 \pm 3^{\circ} \mathrm{C}\right)$ with aqueous solutions of glycerol (anhydrous, purity $\geqslant 99 \%$, MERCK) at glycerol concentrations of 40 or $60 \% \mathrm{~m} / \mathrm{m}$, respectively, with kinematic viscosities of $v^{25^{\circ} \mathrm{C}}=2.85 \times 10^{-6} \mathrm{~m}^{2} / \mathrm{s}(40 \% \mathrm{~m} / \mathrm{m})$ and $v^{25^{\circ} \mathrm{C}}=$ $7.55 \times 10^{-6} \mathrm{~m}^{2} / \mathrm{s}(60 \% \mathrm{~m} / \mathrm{m})-$ Chen and Pearlstein, 1987. The unit used for the experiments is depicted in Fig. 2. Both bottom and head of the Taylor-vortex device were made of stainless steel. The cylindrical stator was manufactured of transparent acrylic glass. Two ribbed rotors A and B as well as a cylindrical rotor $C$ were used for this study, all of which were of aluminium. Both of the ribbed rotors were segmented into 20 mixing cells of equal size, but the heights of the ribs $\left(h_{\text {rib }}\right)$ and, accordingly, the lengths of the rotors were different. All dimensions of the reactor configurations investigated are summarised in Table 1 .

The rotors were driven by an electronic drive control with integrated torque monitoring (RZR 2102 Control, HEIDOLPH) in the range of $12-700 \mathrm{rpm}(40<\mathrm{Ta}<2500)$. A permanent magnetic coupling (Minex-S SA 60/8, KTR) was attached to seal the connection between rotor and drive. The liquid was fed through a conus into

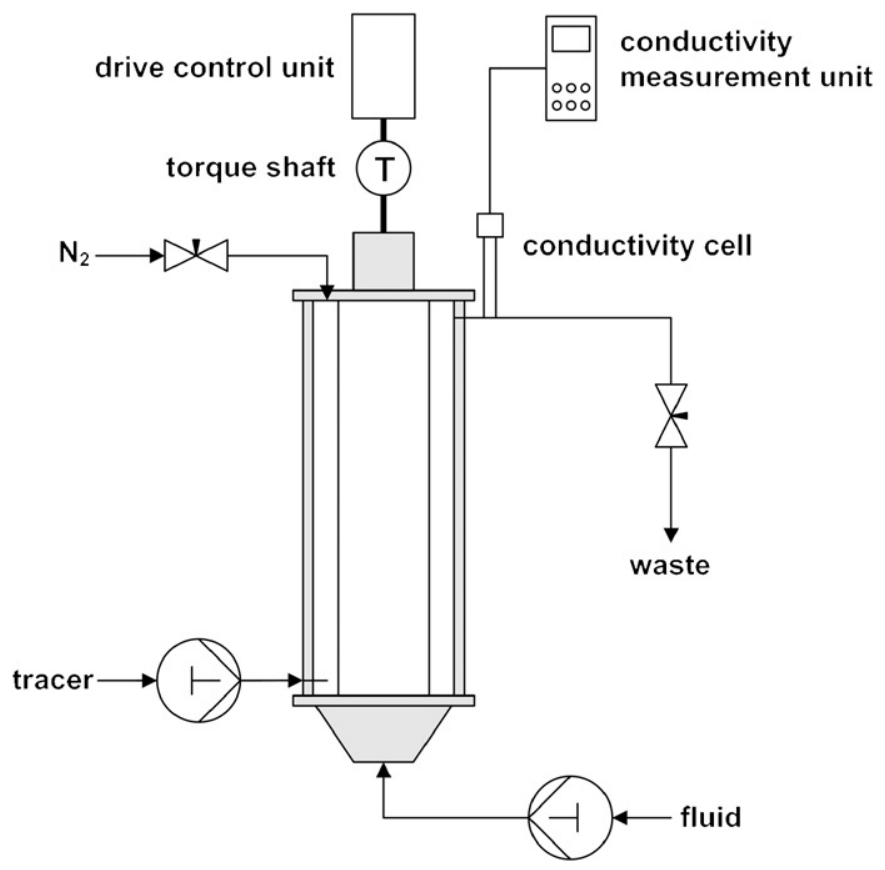

Fig. 2. Experimental set-up. 
Table 1

Rotor dimensions in $\mathrm{mm}$ and corresponding reactor volumes in $\mathrm{mL}$

\begin{tabular}{lrrc}
\hline & A & B & C \\
\hline$r_{0}$ & 40.0 & 40.0 & 40.0 \\
$r_{i}$ & 35.0 & 35.0 & 35.0 \\
$r_{\text {rib }}$ & 39.0 & 39.0 & - \\
$h_{\text {rib }}$ & 8.0 & 2.0 & - \\
$h_{\text {cell }}$ & 10.0 & 10.0 & - \\
$L$ & 352.0 & 238.0 & 280.0 \\
$V_{r}$ & 260.7 & 232.4 & 329.9 \\
\hline
\end{tabular}

The symbols are defined in Fig. 1.

the reactor bottom. The flow rates ranging from 3.5 to $22.0 \mathrm{~mL} / \mathrm{min}$ $(0.03<R e<0.51)$ were generated by means of an electronically controlled rotary piston pump (MCP-CPF Control, ISMATEC; pump head QP Q1.CSC-W). The head of the reactor was pressurised with nitrogen in order to adjust a constant level of the liquid.

\subsection{RTD measurements}

Residence time distributions (RTD) were obtained from tracer experiments using potassium chloride $(\mathrm{KCl}$, purity $\geqslant 99.5 \%$, MERCK) dissolved in demineralised water $(25 \% \mathrm{~m} / \mathrm{m})$ as a tracer, which was pulse-injected $(300 \mu \mathrm{L}$ in $0.5 \mathrm{~s})$ by means of a second rotary piston pump (MCP-CPF Control, ISMATEC; pump head QP Q2.CSC-W). The molecular diffusion coefficients for $\mathrm{KCl}$ in glycerol-water solutions (Hosokawa et al., 1975) varied between $D_{\mathrm{mol}}^{25^{\circ} \mathrm{C}}=8.7 \times 10^{-10} \mathrm{~m}^{2} / \mathrm{s}$ $(40 \% \mathrm{~m} / \mathrm{m}$ glycerol $)$ and $D_{\mathrm{mol}}^{25^{\circ} \mathrm{C}}=5.7 \times 10^{-10} \mathrm{~m}^{2} / \mathrm{s}(60 \% \mathrm{~m} / \mathrm{m}$ glycerol). Irrespective of the rotor type used, the tracer was injected into the core of the first vortex formed at the reactor bottom. The conductivity signal, here proportional to the tracer concentration $c(t)$, was continuously measured directly at the reactor outlet using a flowthrough conductivity cell (TetraCon 325/Cond 340i, WTW). To avert adulteration of the RTD, the conductivity cell was filled with glass beads $(\varnothing \approx 1 \mathrm{~mm}$ ) such that the effective volume was $2 \mathrm{~mL}$, only. The RTD is reported as normalised tracer concentration $E_{\Theta}$ over the normalised time $\Theta$.

$E_{\Theta}=\frac{V_{r} \cdot c(t)}{\dot{V} \cdot \int_{0}^{\infty} c(t) \mathrm{d} t}$

$\Theta=\frac{\dot{V} \cdot t}{V_{r}}$

\subsection{Specific input of mechanical power}

The input of mechanical power can be monitored through the torque of the rotor and is a measure for the intensity of the overall mixing. The reference point of each rotor was set by measuring the torque in the absence of liquid after rotating at $400 \mathrm{rpm}$ for $10 \mathrm{~min}$. When filled, the rotor torque was recorded at the desired speed over a time range of $10 \mathrm{~min}$. The arithmetic average value of the torque $T$ was then used to calculate the specific power input $\varepsilon$ according to the following equation:

$\varepsilon=\frac{P}{\rho \cdot V_{r}}=\frac{T \cdot \omega}{\rho \cdot V_{r}}$

A dimensional analysis shows that the power consumption of a Taylor-vortex rotor is dependent on rotor speed, fluid viscosity, fluid density and rotor dimensions. Accordingly, a dimensionless specific power input $\Pi$ can be defined to characterise the overall extent of micromixing.

$\Pi=\frac{\varepsilon^{0.5} \cdot r_{i}^{2}}{v^{1.5}}$

\subsection{Flow visualisation}

The aqueous solutions of glycerol were transformed into rheoscopic fluids by adding $0.5 \mathrm{~g} / \mathrm{L}$ of Iriodin ${ }^{\circledR}$ (Iriodin ${ }^{\circledR}$ silver-white pigment, MERCK). This substance consists of small, light reflecting slabs $(\emptyset<25 \mu \mathrm{m})$, which arrange themselves along streamlines due to the viscous forces.

\section{Modelling of the mass transport}

Macromixing is described with the dimensionless dispersion number $\mathbf{D}$, which equals the ratio of dispersive to convective intervortex mass transfer. Accordingly, a low extent of macromixing is indicated by a small value of $\mathbf{D}$.

$\mathbf{D}=\frac{D_{\mathrm{ax}}}{u_{\mathrm{ax}} \cdot L}$

The dispersion numbers $\mathbf{D}$ were calculated from the inter-vortex mass transfer parameters which, in turn, were deduced from numerical fits (least square method) of the RTD. Several phenomenological mass transport models based on the multi-zone model were evaluated. As will be shown, however, one-zone models turned out to be sufficient, in which a toroidal vortex was represented as one perfectly mixed zone. This simplification was possible because the long reactor residence times applied here (up to $60 \mathrm{~min}$ ) ensured adequate intra-vortex mixing.

\subsection{One-zone model for the cylindrical rotor}

As shown in Fig. 3, the annulus was divided into perfectly mixed zones with a volume $V_{V}$ and a diameter $d=r_{0}-r_{i}$, which move downstream with a drift velocity $u_{d}$. Bypass was not incorporated as a separate section but rather indirectly by considering a vortex slowdown, which is caused by a bypass stream. Hence, the vortex drift velocity $u_{d}$ was a fraction $f$ of the superficial velocity $u_{\mathrm{ax}}$. Mass transfer between the vortices via the annular contact surface $A_{V}$ was expressed as a mass transfer coefficient $\beta_{V}$, which also included the effect of a bypass stream on the inter-vortex mass transport.

The mass balance of the system results in the following system of coupled ordinary differential equations (ode):

vortex 1

$\frac{\mathrm{d} c_{1}}{\mathrm{~d} t}=\frac{A_{V} \cdot \beta_{V}}{V_{V}} \cdot\left(c_{2}-c_{1}\right)$

vortex $n$

$\frac{\mathrm{d} c_{n}}{\mathrm{~d} t}=\frac{A_{V} \cdot \beta_{V}}{V_{V}} \cdot\left(c_{n-1}-2 \cdot c_{n}+c_{n+1}\right)$

vortex $N$

$\frac{\mathrm{d} c_{N}}{\mathrm{~d} t}=\frac{A_{V} \cdot \beta_{V}}{V_{V}} \cdot\left(c_{N-1}-c_{N}\right)$

The incremental calculation of the dispersion started with the initial $(t=0)$ tracer concentration $c_{1}=c_{0} ; c_{n}=c_{N}=0 \mathrm{~mol} / \mathrm{m}^{3}$. Then the 


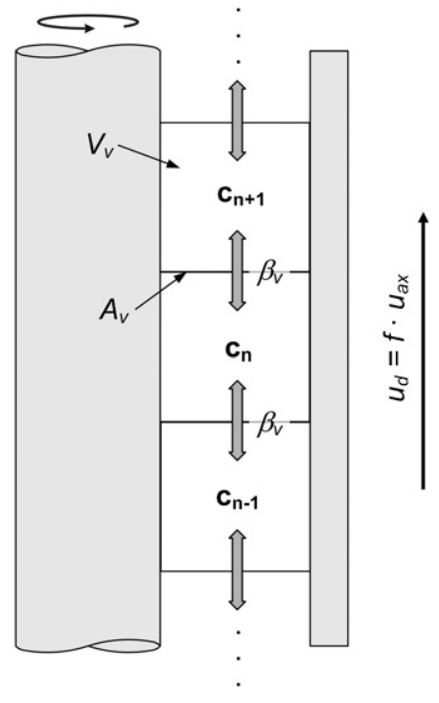

Fig. 3. Scheme of the inter-vortex mass transport in a Taylor-vortex reactor with cylindrical rotor.

vortices were virtually forced to jump one mixing zone downstream, whereby the last $N$-th vortex leaved the reactor with the outlet concentration $c_{N}$, and a fresh vortex came in with $c_{1}=0 \mathrm{~mol} / \mathrm{m}^{3}$. The incremental time between the jumps is $\Delta t=d / u_{d}$. This routine was repeated until the outlet concentration reached zero. The intermediate outlet concentrations $c_{N}(t)$ were used to solve Eq. (3) and to plot the modelled RTD. The overall dimensionless dispersion number $\mathbf{D}$ for the system with cylindrical rotor was obtained from the fitted mass transfer parameters using Eqs. (11) and (12).

$\frac{1}{k_{c}}=\frac{1}{\beta_{V}}+\frac{1}{\beta_{V}}$

$\mathbf{D}=\frac{D_{\mathrm{ax}}}{u_{d} \cdot L}=\frac{k_{c} \cdot d}{f \cdot u_{\mathrm{ax}} \cdot L}$

\subsection{One-zone model for the ribbed rotors}

The transport model for the ribbed rotors had to be slightly adapted. Flow visualisation showed that pairs of two vortices turning in opposite directions are formed, building an immobilised mixing cell (index $n$ ). Each vortex pair was modelled as a perfectly mixed up-zone (index $u$ ) and down-zone (index $d$ ) (volume $V_{V}$; diameter $d$ ). The volume of the annular space between rib and stator was split and added to the volumes of the adjacent vortices (Fig. 4). Mass transfer inside each vortex pair via the annular contact surface $A_{i}$ was specified by the mass transfer coefficient $\beta_{i}$. Between two mixing cells separated by a rib, mass transport via the annular contact surface $A_{0}$ was represented by the mass transfer coefficient $\beta_{0}$. Additional convective mass transport occurred here by axial flow.

The mass balance of each vortex yielded the following coupled ode-system with the initial condition $(t=0): c_{1, d}=c_{0} ; c_{1, u}=\cdots=$ $c_{N, u}=0 \mathrm{~mol} / \mathrm{m}^{3}$.

vortex $1, d$

$\frac{\mathrm{d} c_{1, d}}{\mathrm{~d} t}=\frac{A_{i} \cdot \beta_{i}}{V_{V}} \cdot\left(c_{1, u}-c_{1, d}\right)$

vortex $1, u$

$$
\begin{aligned}
\frac{\mathrm{d} c_{1, u}}{\mathrm{~d} t}= & -\frac{\dot{V}}{V_{V}} \cdot c_{1, u}-\frac{A_{i} \cdot \beta_{i}}{V_{V}} \cdot\left(c_{1, u}-c_{1, d}\right) \\
& -\frac{A_{o} \cdot \beta_{0}}{V_{V}} \cdot\left(c_{1, u}-c_{2, d}\right)
\end{aligned}
$$

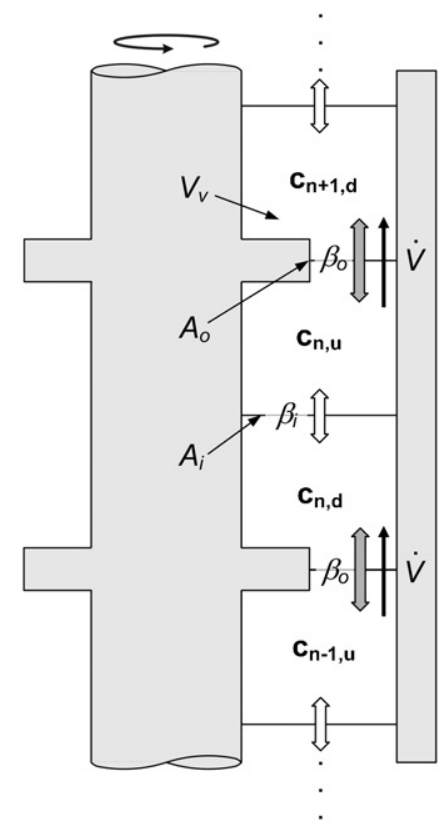

Fig. 4. Scheme of the inter-vortex mass transport in a Taylor-vortex reactor with ribbed rotor.

vortex $n, d$

$$
\begin{aligned}
\frac{\mathrm{d} c_{n, d}}{\mathrm{~d} t}= & \frac{\dot{V}}{V_{V}} \cdot c_{n-1, u}-\frac{A_{i} \cdot \beta_{i}}{V_{V}} \cdot\left(c_{n, d}-c_{n, u}\right) \\
& -\frac{A_{0} \cdot \beta_{0}}{V_{V}} \cdot\left(c_{n, d}-c_{n-1, u}\right)
\end{aligned}
$$

vortex $n, u$

$$
\begin{aligned}
\frac{\mathrm{d} c_{n, u}}{\mathrm{~d} t}= & -\frac{\dot{V}}{V_{V}} \cdot c_{n, u}-\frac{A_{i} \cdot \beta_{i}}{V_{V}} \cdot\left(c_{n, u}-c_{n, d}\right) \\
& -\frac{A_{o} \cdot \beta_{0}}{V_{V}} \cdot\left(c_{n, u}-c_{n+1, d}\right)
\end{aligned}
$$

$\operatorname{vortex} N, d$

$$
\begin{aligned}
\frac{\mathrm{d} c_{N, d}}{\mathrm{~d} t}= & \frac{\dot{V}}{V_{V}} \cdot c_{N-1, u}-\frac{A_{i} \cdot \beta_{i}}{V_{V}} \cdot\left(c_{N, d}-c_{N, u}\right) \\
& -\frac{A_{0} \cdot \beta_{0}}{V_{V}} \cdot\left(c_{N, d}-c_{N-1, u}\right)
\end{aligned}
$$

vortex $N, u$

$$
\frac{\mathrm{d} c_{N, u}}{\mathrm{~d} t}=-\frac{\dot{V}}{V_{V}} \cdot c_{N, u}-\frac{A_{i} \cdot \beta_{i}}{V_{V}} \cdot\left(c_{N, u}-c_{N, d}\right)
$$

The overall dispersion number $\mathbf{D}$ of the Taylor-vortex reactor with ribbed rotor was calculated from the fitted mass transfer parameters. If each of the 20 mixing cells would perform as an ideally stirred tank, the theoretical minimum dispersion number of the reactor $\mathbf{D}_{\text {STC }}$ would be that of a stirred tank cascade (STC) with 20 vessels $\left(\mathbf{D}_{\mathrm{STC}}=\right.$ $2.566 \times 10^{-2}$ ). The dispersion numbers of the real systems are higher and can be calculated using Eq. (20).

$$
\begin{aligned}
& \frac{1}{k_{r}}=\frac{1}{\beta_{0}}+\frac{1}{\beta_{i}}+\frac{1}{\beta_{0}} \\
& \mathbf{D}=\mathbf{D}_{\mathrm{STC}}+\frac{D_{\mathrm{ax}}}{u_{\mathrm{ax}} \cdot L}=\mathbf{D}_{\mathrm{STC}}+\frac{k_{r} \cdot 2 d}{u_{\mathrm{ax}} \cdot L}
\end{aligned}
$$




\section{Results and discussion}

\subsection{Flow visualisation}

Fig. 5 shows the flow patterns caused with the cylindrical rotor $\mathrm{C}$ at four different $\mathrm{Ta}$ numbers. The toroidal vortices always moved downstream in stack without helical deformation. Within the range of experimental conditions under investigation $(0.03<R e<0.51)$, all detected flow transitions were independent on the Re number.

The first flow transition (CF to LTVF) occurred near $T a=80$. This value and the negligible dependence on $R e$ are in accordance with transition points theoretically predicted by Recktenwald et al. (1993) of $T a_{c}=81.40$ at $R e=0.03$ and $T a_{c}=81.42$ at $R e=0.51$. The LTVF regime was stable only in a narrow range of $T a$ numbers between 80 and 130. The WVF regime was observed up to $T a=600(130<T a<600)$, followed by TVF flow at $T a>600$.

As compared to the cylindrical rotor $\mathrm{C}$, the ribbed rotors $\mathrm{A}\left(h_{\mathrm{rib}}=\right.$ $8 \mathrm{~mm})$ and $\mathrm{B}\left(h_{\mathrm{rib}}=2 \mathrm{~mm}\right)$ caused entirely different flow regimes. The three typical patterns obtained with rotor A are shown in Fig. 6. Irrespective of the $\mathrm{Ta}$ - and $\mathrm{Re}$ numbers adjusted, there were always two counter-rotating toroidal vortices (vortex-pair) formed between two ribs in a mixing cell (light bands in Fig. 6). Again, all flow patterns and transitions were independent of $\operatorname{Re}(0.03<\operatorname{Re}<0.51)$.

The observed changes with increasing Ta do not refer to the mixing cells but rather to the narrow annular gaps between ribs and stator (dark regions in Fig. 6). There, laminar shear flow (Couette flow) existed at $0<T a<750$, denoted as primary laminar vortex flow, PLVF. The first flow transition appeared near $T a=750$, transforming the shear flow into laminar Taylor vortices $(750<T a<1200$, sec-
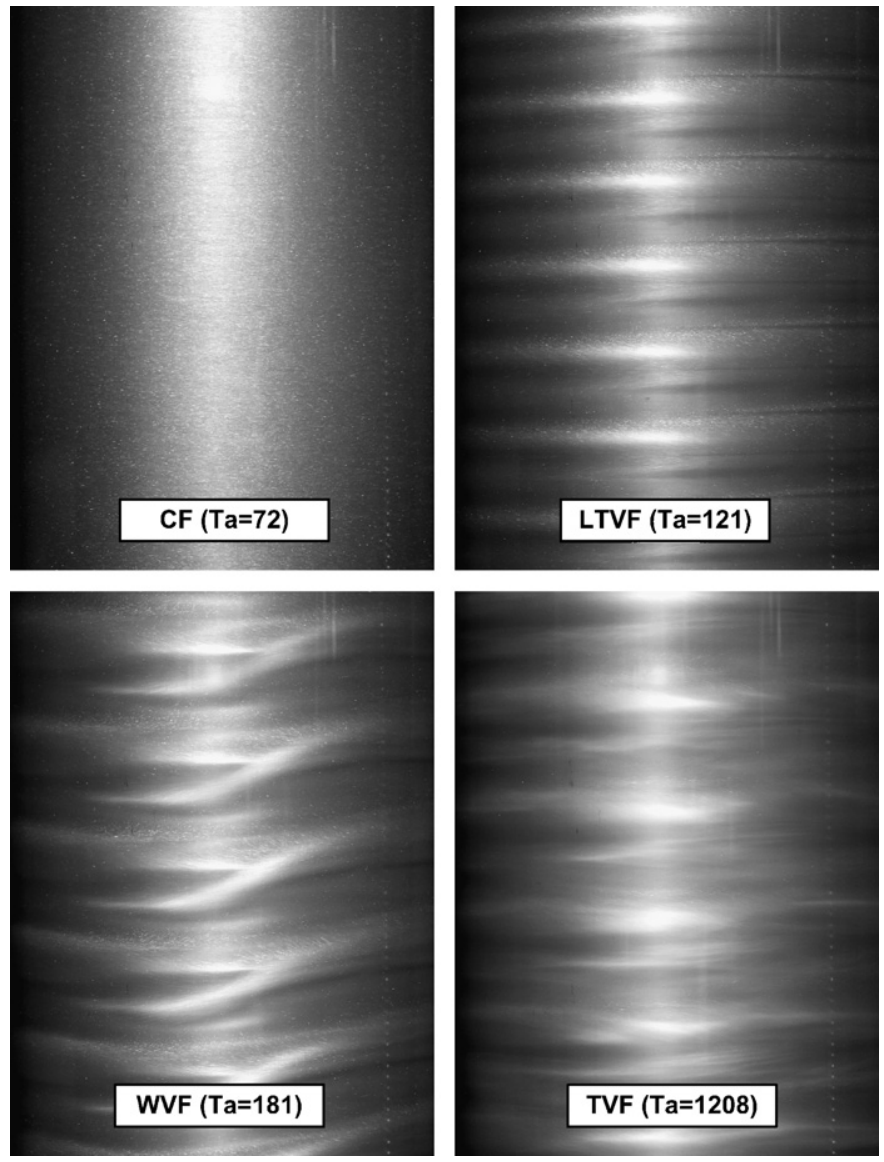

Fig. 5. Front view of the continuous flow Taylor-vortex reactor with cylindrical rotor $\mathrm{C}$ showing the typical flow patterns ( $R e=0.13$; rheoscopic fluid: $0.5 \mathrm{~g} / \mathrm{L}$ Iriodin ${ }^{\circledR}$ silver-white pigment dispersed in $40 \% \mathrm{~m} / \mathrm{m}$ glycerol-water).
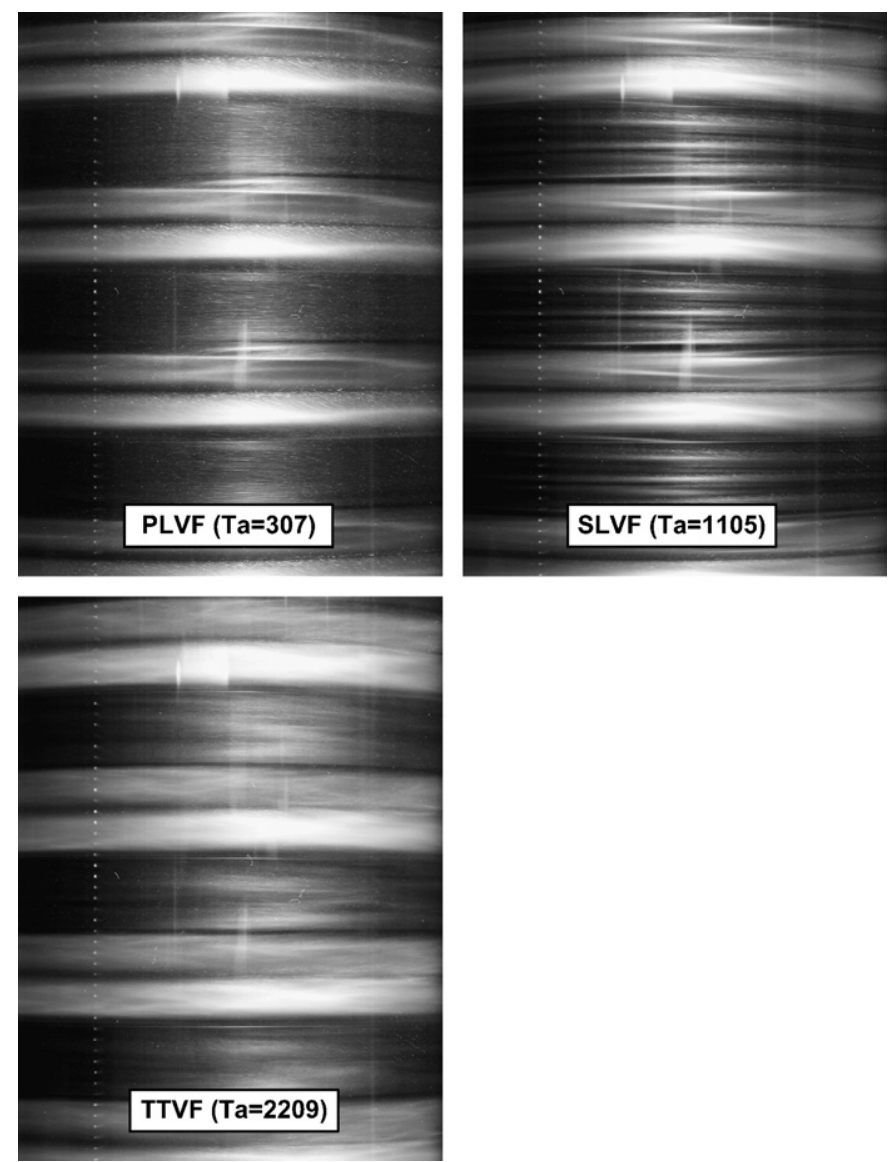

Fig. 6. Front view of the continuous flow Taylor-vortex reactor with ribbed rotor A showing typical flow patterns $\left(h_{\text {rib }}=8 \mathrm{~mm}\right.$; $R e=0.19$; rheoscopic fluid: $0.5 \mathrm{~g} / \mathrm{L}$ Iriodin ${ }^{\mathrm{B}}$ silver-white pigment dispersed in $40 \% \mathrm{~m} / \mathrm{m}$ glycerol-water).

ond laminar vortex flow, SLVF). A further increase in the Ta number caused turbulence $(1200<\mathrm{Ta}$, third turbulent vortex flow, TTVF).

In spite of the superimposed axial flow, the vortex-pairs in the mixing cells did not move downstream; they were immobilised between the ribs. A comparison of the photographs in Figs. 5 and 6 clearly shows that the ribs also have a stabilising effect. No azimuthal oscillations were visible as in the WVF regime of the cylindrical rotor $C$, and no deformation or overlapping of the vortices within a mixing cell was noticed. In addition, the onset of turbulent structures shifted to considerably higher Ta numbers (cylindrical rotor $C$, TVF: $600<$ Ta; ribbed rotors $A$ and $B$, TTVF: $1200<T a)$.

\subsection{Validation of the mass transport models}

Fig. 7 shows the normalised tracer RTD obtained from tracer experiments (symbols) together with the plots resulting from the transport models. For both rotor types, a good fit of the experimental data was achieved, indicating that the two one-zone models developed are suitable to describe the mass transport.

Fig. 7 also allows a first comparison of the macromixing characteristics of the two rotor types because the hydrodynamic conditions ( $\mathrm{Ta}, \mathrm{Re}$ ) applied during data acquisition were similar. Obviously, the RTD in the reactor with conventional cylindrical rotor is much broader than the RTD obtained with the ribbed rotor. Hence, the segmentation of the annulus by ribs has the hoped-for effect, i.e. the reduction of macromixing in the Taylor-vortex reactor. A more detailed inspection follows in Sections 4.3-4.5. 


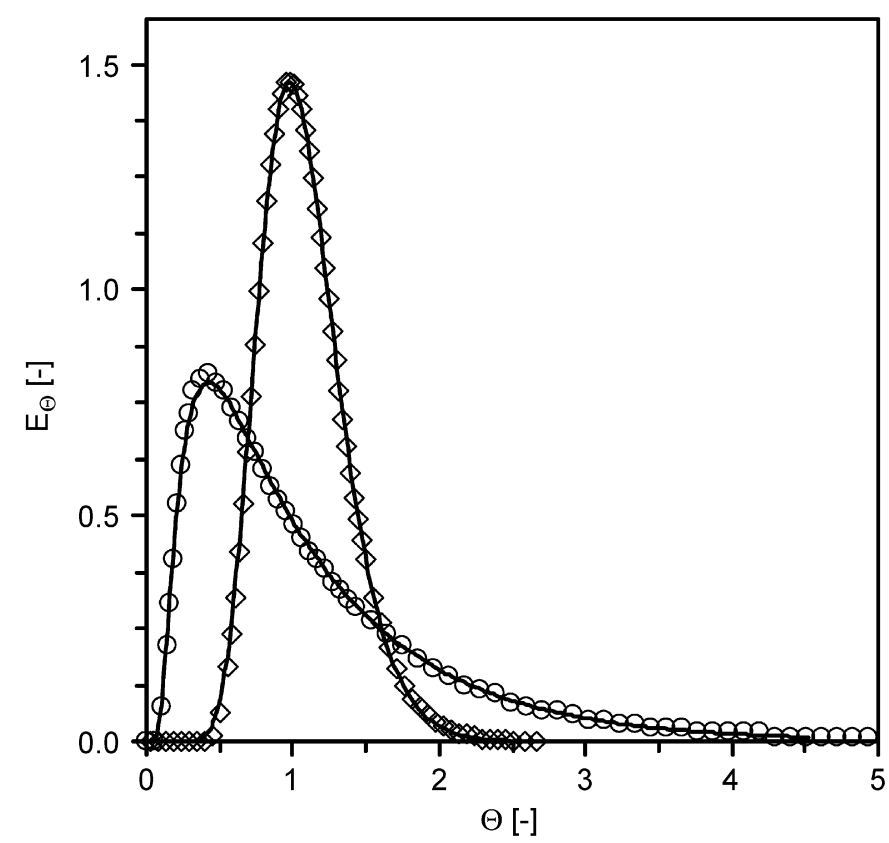

Fig. 7. Comparison of calculated (-) and experimental RTD upon use of the cylindrical rotor $\mathrm{C}(\bigcirc)$ and the ribbed rotor $\mathrm{A}(\diamond) ;(60 \% \mathrm{~m} / \mathrm{m}$ glycerol-water; cylindrical rotor $C: T a=447, R e=0.19, \mathbf{D}=3.1 \times 10^{-1}$; ribbed rotor $A: h_{\text {rib }}=8 \mathrm{~mm}, T a=503, R e=0.16$, $\left.\mathbf{D}=4.3 \times 10^{-2}\right)$.

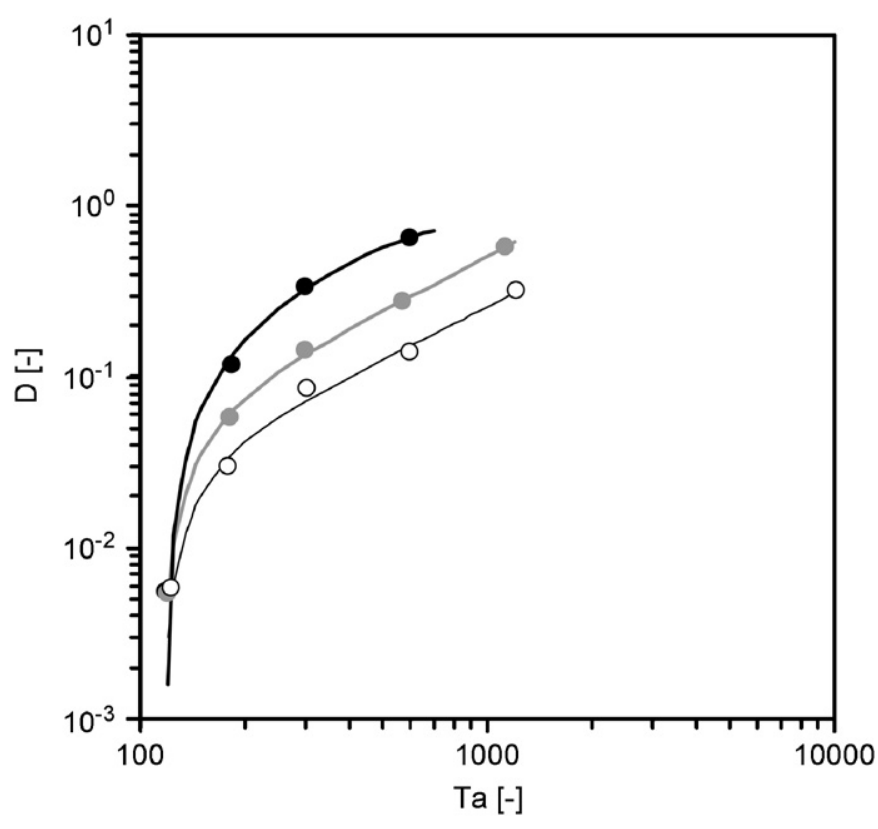

Fig. 8. Dimensionless dispersion numbers $\mathbf{D}$ over $\mathrm{Ta}$ obtained with the cylindrical rotor $\mathrm{C}$ and $40 \% \mathrm{~m} / \mathrm{m}$ glycerol-water solutions; $R e=0.51(\mathrm{O}), \operatorname{Re}=0.24(\mathrm{O})$ and $R e=0.13$

\subsection{Macromixing in a reactor with cylindrical rotor}

The dispersion numbers determined for the reactor equipped with the cylindrical rotor $C$ are shown in Figs. 8 and 9 for 40 and $60 \% \mathrm{~m} / \mathrm{m}$ glycerol-water solutions, respectively. The $\mathrm{CF}$ regime $(\mathrm{Ta}<80$ ) was not inspected because of its low practical relevance.

The lowest $\mathbf{D}$-values between $10^{-3}$ and $10^{-2}$ refer to the LTVF regime and indicate an overall behaviour close to plug flow. The move into the WVF regime $(130<T a<600)$ is accompanied by a

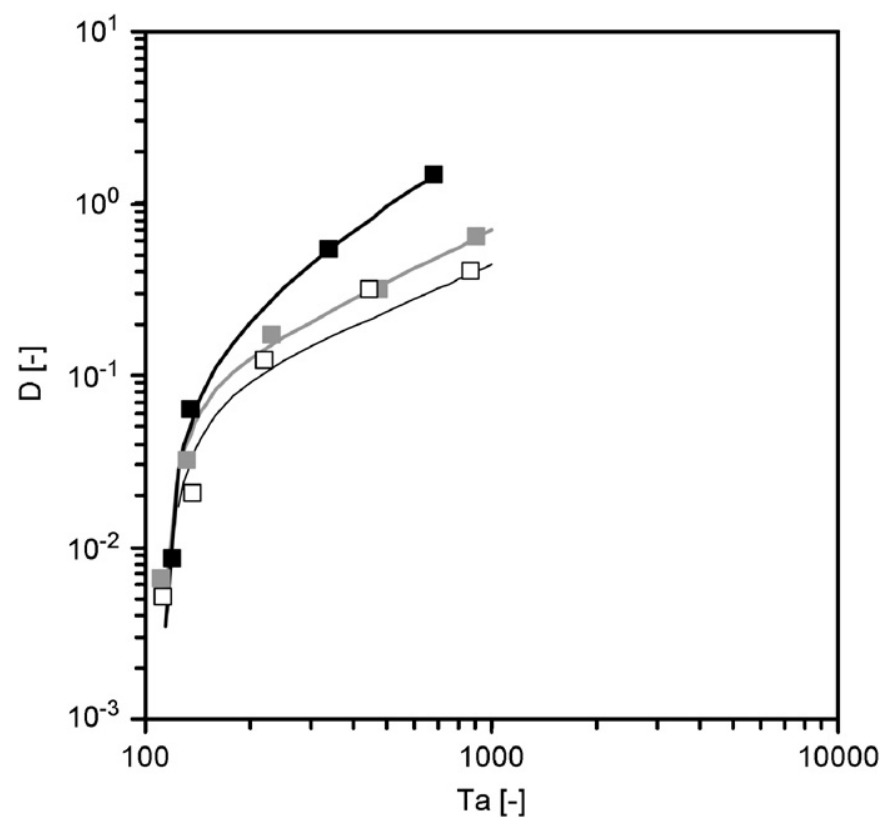

Fig. 9. Dimensionless dispersion numbers $\mathbf{D}$ over Ta obtained with the cylindrical rotor $C$ and $60 \% \mathrm{~m} / \mathrm{m}$ glycerol-water solution; $R e=0.19(\square), R e=0.10$ (口) and $R e=0.05$

pronounced increase in the dispersion numbers. At even higher $\mathrm{Ta}$ numbers, the extent of macromixing approaches a level similar to a stirred tank reactor.

As will be shown (Eq. (21)), the dispersion coefficient $D_{\mathrm{ax}}$ is only a weak function of Re. The dependence of the dispersion number $\mathbf{D}$ on Re as visible in Figs. 8 and 9 basically originated from the variation of the superficial velocity $u_{\mathrm{ax}}$.

We compared our measured dispersion coefficients $D_{\mathrm{ax}}$ with literature data. By means of tracer experiments, Moore and Cooney (1995) investigated inter-vortex mass transport in a continuous flow Taylor-vortex device with plenty of cylindrical geometries $\quad\left(19.05 \mathrm{~mm}<r_{i}<26.75 \mathrm{~mm} ; 24.50 \mathrm{~mm}<r_{0}<31.15 \mathrm{~mm}\right.$; $70 \mathrm{~mm}<L<252 \mathrm{~mm})$ in a wide hydrodynamic range $(200<\mathrm{Ta}<$ $20000 ; 0.25<R e<15$ ). They deduced a mass transfer correlation with water $\left(v^{25^{\circ} \mathrm{C}}=1.0 \times 10^{-6} \mathrm{~m}^{2} / \mathrm{s}\right)$ as a working fluid and confirmed viscosity dependence only expressed within $\mathrm{Ta}$ and $\mathrm{Re}$ by two additional experiments with $25 \% \mathrm{~m} / \mathrm{m}\left(v^{25^{\circ} \mathrm{C}}=1.8 \times 10^{-6} \mathrm{~m}^{2} / \mathrm{s}\right)$ and $45 \% \mathrm{~m} / \mathrm{m}\left(v^{25^{\circ} \mathrm{C}}=3.7 \times 10^{-6} \mathrm{~m}^{2} / \mathrm{s}\right)$ glycerol-water solutions. Furthermore, they verified diffusion independence of the macromixing at their experimental conditions by using blue dextran $\left(D_{\mathrm{mol}}=8 \times 10^{-12} \mathrm{~m}^{2} / \mathrm{s}\right)$ and sodium benzoate $\left(D_{\mathrm{mol}}=8 \times 10^{-10} \mathrm{~m}^{2} / \mathrm{s}\right)$ as tracers.

The mass transfer correlation by Moore and Cooney (Eq. (21)) shows that inter-vortex mass transport is mainly affected by $\mathrm{Ta}$, less by $R e$ and is independent of diffusion.

$D_{\mathrm{ax}} \cdot\left(\frac{d}{r_{i}}\right)^{0.28} \cdot(2 \cdot R e)^{-0.17}=7.2 \times 10^{-9} \mathrm{~m}^{2} / \mathrm{s} \cdot \mathrm{Ta}^{1.05}$

This correlation is plotted in Fig. 10 together with our experimental data taken with the cylindrical rotor $\mathrm{C}$.

In case of the solution containing $40 \% \mathrm{~m} / \mathrm{m}\left(v^{25^{\circ} \mathrm{C}}=2.85 \times\right.$ $10^{-6} \mathrm{~m}^{2} / \mathrm{s}$ ) glycerol, a good agreement between correlation and experimental data can be established. However, the data obtained with the solution of higher glycerol concentration do not fit well. They show that a significantly larger viscosity $\left(v^{25^{\circ} \mathrm{C}}=7.55 \times 10^{-6} \mathrm{~m}^{2} / \mathrm{s}\right)$ causes a slight enhancement of the inter-vortex mass transfer. The mass transfer correlation by Moore and Cooney is adequate only in a low viscosity range from $v^{25^{\circ} \mathrm{C}}=1.0 \times 10^{-6}$ till $3.7 \times 10^{-6} \mathrm{~m}^{2} / \mathrm{s}$. 


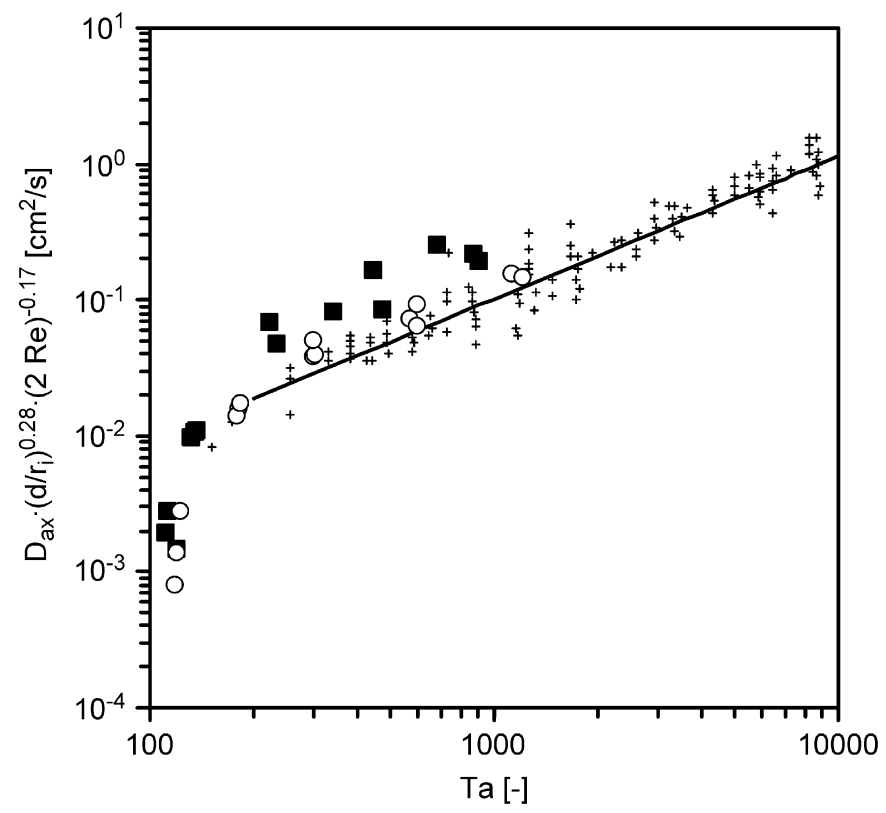

Fig. 10. Inter-vortex mass transfer correlation $(-)$ and data $(+)$ given by Moore and Cooney (1995) compared with experimental data obtained with the cylindrical rotor $\mathrm{C}$ and with $40 \% \mathrm{~m} / \mathrm{m}(\mathrm{O})$ and $60 \% \mathrm{~m} / \mathrm{m}$ (口) glycerol-water.

The viscosity influence on axial dispersion seems to be stronger than just expressed within $\mathrm{Ta}$ and Re. In flow regimes governed by convection (WTV, TVF), higher viscosities require a higher speed of rotation in order to maintain a constant Ta number. As a consequence, this acceleration of the vortex streamline velocity may enhance the convective mass transfer between vortices.

In any case, the experimental data deviate at $T a<200$, and the magnitudes of the dispersion coefficients $D_{\text {ax }}$ approach values characteristic for diffusion. Obviously, diffusion is more dominant at low Ta numbers, i.e. in the LTVF regime, whereas the correlation was developed for convective mass transfer only.

\subsection{Macromixing in a reactor with ribbed rotor}

While macromixing is continuously enhanced with increasing rotor speed in conventional Taylor-vortex devices, the effect of ribbed rotors is different. The data in Figs. 11-13 referring to two different rib heights and liquid viscosities show that the dispersion passes a minimum. This minimum coincides with the SLVF regime.

Within the same hydrodynamic range, the dispersion numbers $\mathbf{D}$ should be independent of the fluid used. The comparison of Figs. 11 and 12 , however, indicates slight differences in that the minima of the dispersion are more pronounced when the liquid with the higher glycerol concentration was used. In this case, even the minimum dispersion in the Taylor-vortex reactor exhibiting 20 mixing cells approaches the theoretical dispersion minimum of an ideal cascade of 20 stirred vessels (dotted lines in Fig. 12). We ascribe this beneficial effect of the higher glycerol concentration to the reduction of the diffusional contribution. The diffusion coefficients of the tracer are $D_{\mathrm{mol}}^{25^{\circ} \mathrm{C}}=8.7 \times 10^{-10} \mathrm{~m}^{2} / \mathrm{s}$ in $40 \% \mathrm{~m} / \mathrm{m}$ glycerol-water solution and $D_{\mathrm{mol}}^{25^{\circ} \mathrm{C}}=5.7 \times 10^{-10} \mathrm{~m}^{2} / \mathrm{s}$ in $60 \% \mathrm{~m} / \mathrm{m}$.

By definition (Eq. (7)), the dispersion number should decrease with increasing axial flow and, hence, increasing Re number. Here, this seems not to hold in the Ta regimes on the left side of the dispersion minima (low Ta). An explanation might be a bypassing of weakly mixed tracer in this regime of less developed vortex flow, and the bypass is more promoted the higher the axial flow is.

The effect of the rib height on macromixing can be elucidated

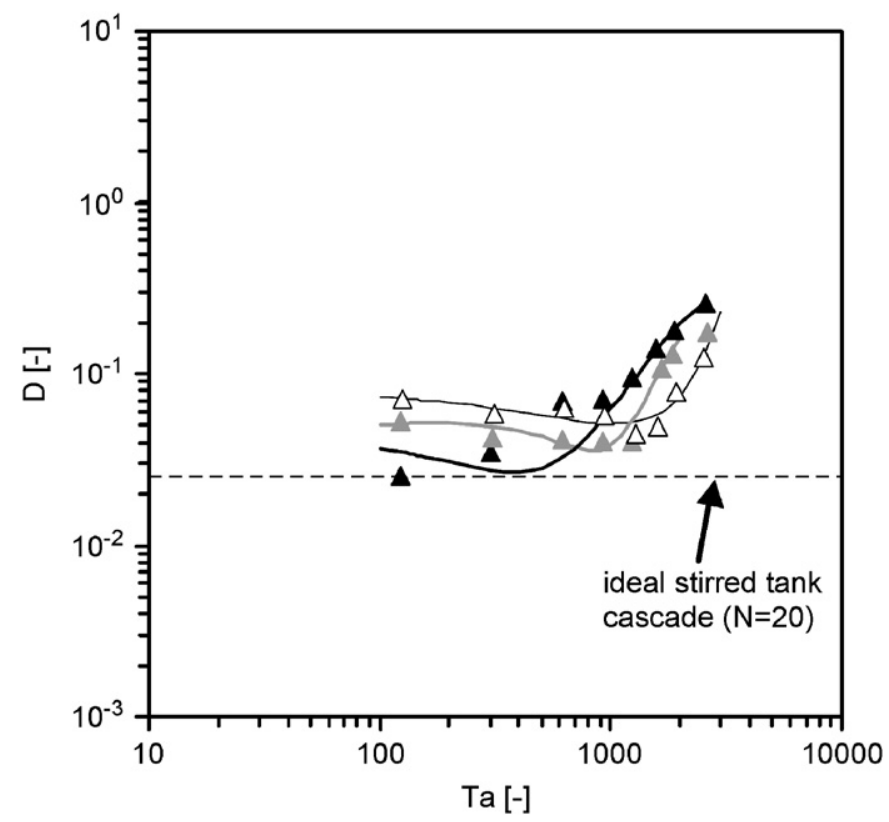

Fig. 11. Dimensionless dispersion numbers $\mathbf{D}$ over Ta obtained with the ribbed rotor $\mathrm{A}\left(h_{\mathrm{rib}}=8 \mathrm{~mm}\right)$ and $40 \% \mathrm{~m} / \mathrm{m}$ glycerol in water; $R e=0.39(\triangle), R e=0.19(\Delta)$ and $\operatorname{Re}=0.10(\boldsymbol{\Delta})$.

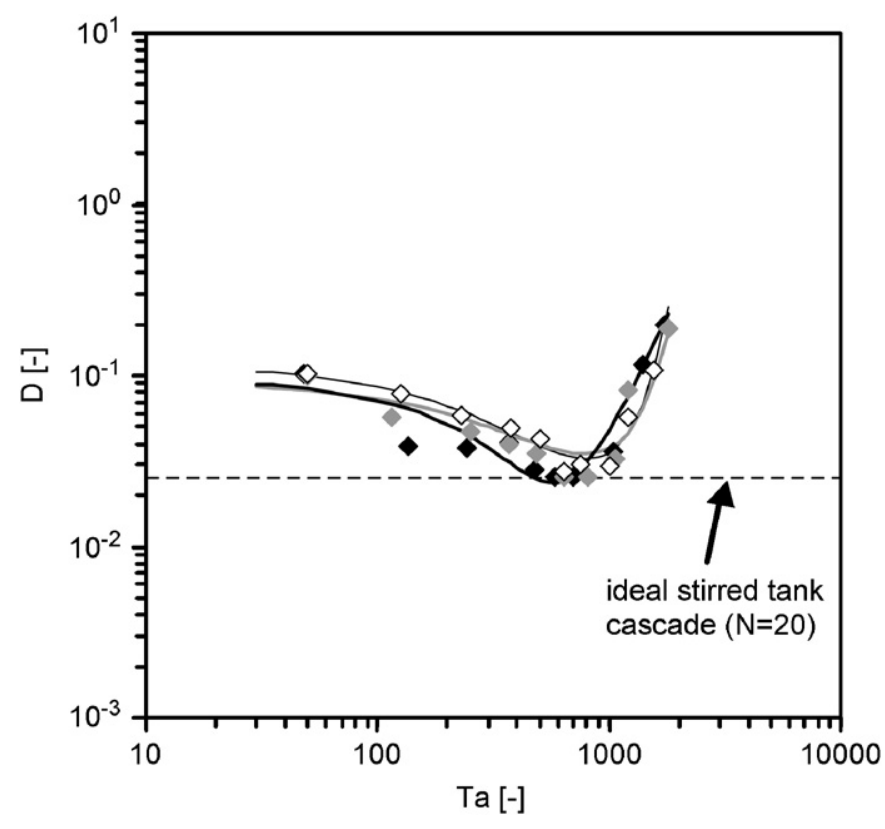

Fig. 12. Dimensionless dispersion numbers $\mathbf{D}$ over Ta obtained with the ribbed rotor $\mathrm{A}\left(h_{\mathrm{rib}}=8 \mathrm{~mm}\right)$ and $60 \% \mathrm{~m} / \mathrm{m}$ glycerol in water; $R e=0.15(\diamond), R e=0.08(\diamond)$ and $R e=0.04(\diamond)$.

by comparing the data in Figs. 12 and 13. The general are similar, but the dispersion numbers $\mathbf{D}$ run on a higher level in case of the rotor with thinner ribs. Hence, macromixing can be reduced more effectively by using rotors with thick ribs.

\subsection{Effect of the rotor shape on macromixing}

The macromixing effects of all rotor geometries inspected in this study can be seen straightaway in Fig. 14. The data selected for the plots were taken at similar Re numbers to enable a meaningful comparison. It is obvious that a Taylor-vortex device with conventional rotor exhibits a low degree of macromixing only at very small $\mathrm{Ta}$ 


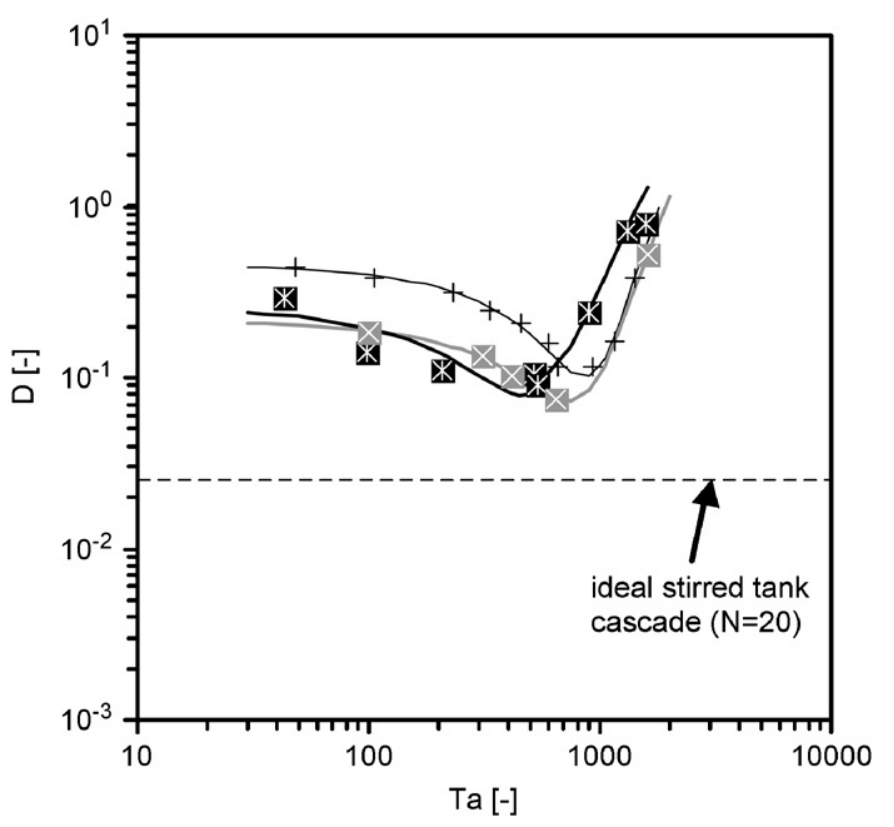

Fig. 13. Dimensionless dispersion numbers $\mathbf{D}$ over $\mathrm{Ta}$ obtained with the ribbed rotor $\mathrm{B}\left(h_{\mathrm{rib}}=2 \mathrm{~mm}\right)$ and $60 \% \mathrm{~m} / \mathrm{m}$ glycerol in water; $R e=0.12(+), R e=0.06(\mathbb{X})$ and $R e=0.03(\mathbb{W})$.

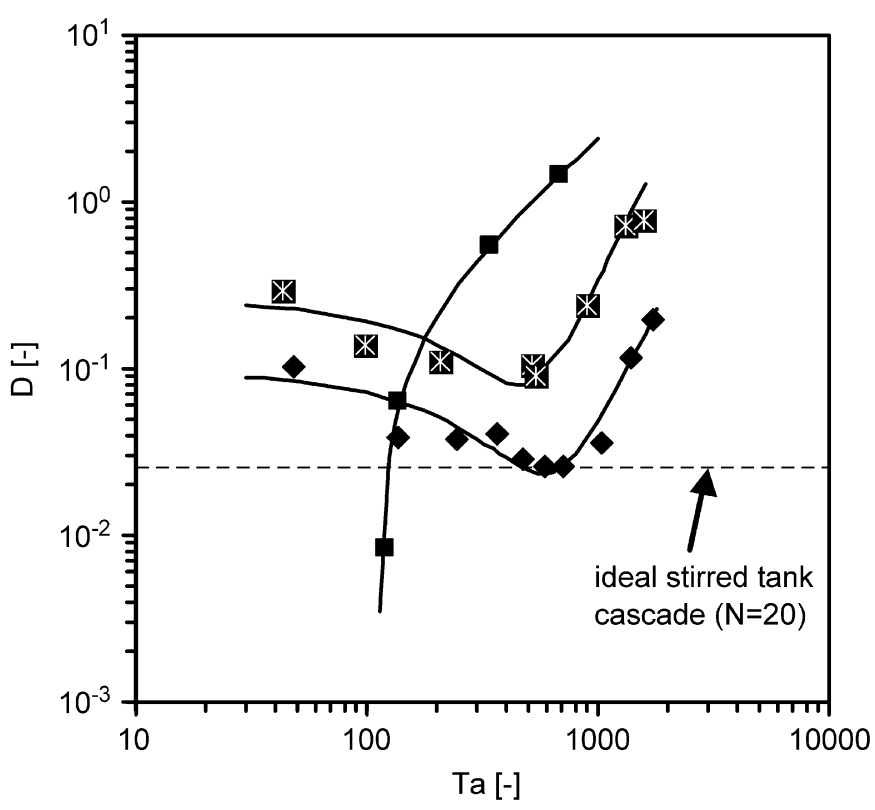

Fig. 14. Comparison of all rotor geometries with respect to the dispersion numbers D over $\mathrm{Ta}$; rotor A $\left(\boldsymbol{\nabla} ; h_{\mathrm{rib}}=8 \mathrm{~mm} ; \operatorname{Re}=0.04\right)$, rotor B $\left(\mathbb{X} ; h_{\mathrm{rib}}=2 \mathrm{~mm} ; R e=0.03\right)$, rotor $\mathrm{C}(\boldsymbol{\square}$; $R e=0.05)$; solution of $60 \% \mathrm{~m} / \mathrm{m}$ glycerol in water.

numbers, and that the macromixing very strongly increases when the laminar flow regime (LTVF) is passed. Ribs on the rotor enable processing at low or moderate macromixing level in a considerably broadened operational window.

\subsection{Micromixing}

Racina and Kind (2006) investigated the specific input of power into a Taylor-vortex devices with different geometries (31.5 $\mathrm{mm}<r_{i}<44.5 \mathrm{~mm}, r_{0}=50 \mathrm{~mm}, L=390 \mathrm{~mm}$ ) and working fluids (water; 20, 30 and $50 \% \mathrm{~m} / \mathrm{m}$ glycerol-water mixtures), and developed a correlation for the rotor-torque applicable in the range

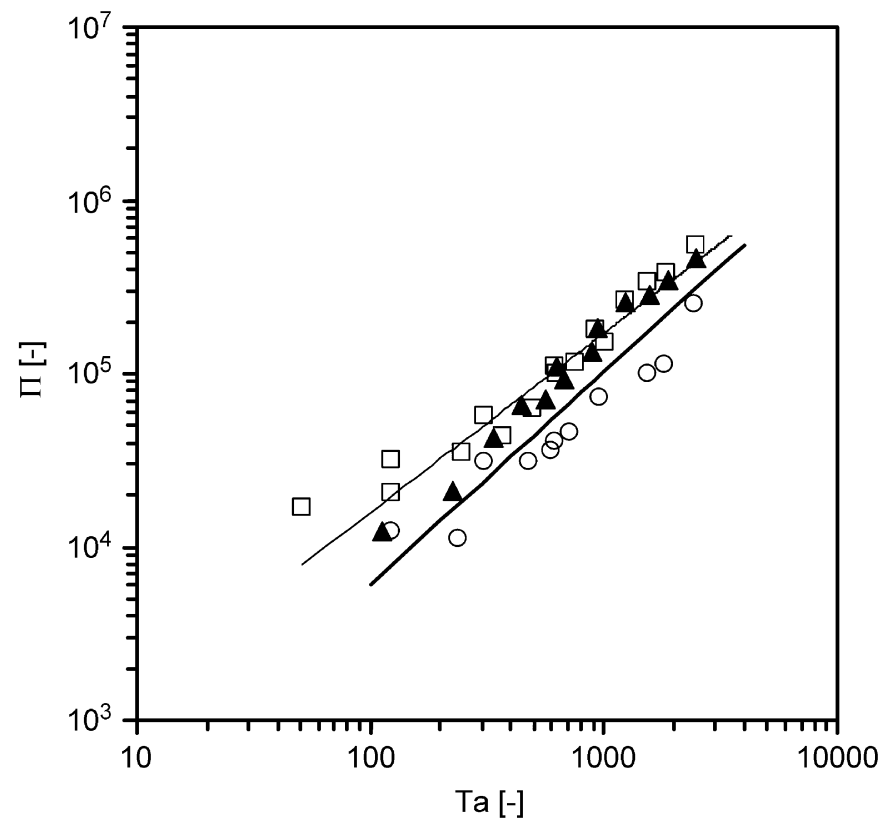

Fig. 15. Dimensionless input of rotor power $\Pi(-)$ according to Racina and Kind (2006), and experimental data obtained with ribbed rotor $\mathrm{A}\left(\square ; h_{\text {rib }}=8 \mathrm{~mm}\right)$, ribbed rotor $\mathrm{B}\left(\boldsymbol{\Delta} ; h_{\mathrm{rib}}=2 \mathrm{~mm}\right)$ and cylindrical rotor $\mathrm{C}(\bigcirc)$ with solutions of 40 and $60 \% \mathrm{~m} / \mathrm{m}$ glycerol concentration.

$800<T a<10000$. From this correlation (extrapolated to $T a=100$ ), the dimensionless integral rotor power input $\Pi$ was calculated according to Eqs. (5) and (6) and plotted in Fig. 15 together with our experimental data obtained with the cylindrical rotor $\mathrm{C}$.

The good agreement between correlation and experimental data confirms that the quality of our measurements is sufficient. As expected, the power input $\Pi$ and, hence, the integral micromixing in the device increase with increasing Ta numbers.

The effect of the rotor shape on the power input is also shown in Fig. 15. The trend line for the ribbed rotors clearly lies above the correlation for the cylindrical rotor. This means that more intense micromixing can be achieved with ribbed rotors. In principle, power transfer with the ribbed rotors could occur in both locations, in the mixing cells and in the small annulus between rib and stator. Both of the ribbed rotors under investigation exhibit the same number and size of mixing cells. Differences refer to the volumes of the small annuli between rib and stator. If the power transfer would contribute significantly, the result should be a higher power input in case of the rotor with thicker ribs. However, Fig. 15 shows that both ribbed rotors perform similarly. We therefore assume that the major part of the integral input of power occurs in the mixing cells. The higher level of micromixing in ribbed as compared to cylindrical rotors can be ascribed to the fact that the fluid is additional radial conveyed by the ribs.

\section{Conclusions}

Flow and mixing characteristics of a continuous flow Taylorvortex device can be altered by modifications of the rotor geometry. In this study, we investigated novel rotors furnished with ribs and compared them with a conventional cylindrical rotor over a wide range of hydrodynamic conditions.

Effects of the ribs visible already with the blank eye are immobilisation and stabilisation of the toroidal vortices, and a shift of the onset of turbulence to higher Ta numbers. The analyses of the mixing characteristics, in particular, demonstrated the benefits of ribbed as compared to cylindrical rotors. The use of ribbed rotors enables the 
intensification of micromixing while at the same time macromixing is reduced considerably at $T a>130$. We anticipate that Taylor-vortex reactors with ribbed rotors can be useful for process intensification in many applications.

\section{Notation}

$A_{i} \quad$ inner annular contact surface of ribbed rotor, $\mathrm{m}^{2}$

$A_{0}$ outer annular contact surface of ribbed rotor, $\mathrm{m}^{2}$

$A_{V} \quad$ annular contact surface of cylindrical rotor, $\mathrm{m}^{2}$

$c(t)$

$c_{n}$

$c_{n, d}$

$c_{n, u}$

$d$

$D_{\text {ax }}$

$D_{\text {mol }}$

D

D $_{\text {STC }}$

$E_{\Theta}$

f

$h_{\text {cell }}$

$h_{\text {rib }}$

$k_{c}$

$k_{r}$

$L$

$N$

$P$

$r_{i}$

$r_{0}$

$r_{\text {rib }}$

Re

$t$

$T$

$\mathrm{Ta}$

$T a_{c}$

$u_{\mathrm{ax}}$

$u_{d}$

$\dot{V}$

$V_{r}$

$V_{v}$

Greek letters

$\beta_{i}$

$\beta_{0}$

$\beta_{v}$

\section{References}

Andereck, C.D., Liu, S.S., Swinney, H.L., 1986. Flow regimes in a circular Couette system with independently rotating cylinders. Journal of Fluid Mechanics 164, 155-183.

Baier, G., Graham, M.D., Lightfood, E.N., 2000. Mass transport in a novel two-fluid Taylor vortex extractor. American Institute of Chemical Engineers Journal 46, 2395-2407.

Campero, R.J., Vigil, R.D., 1997. Axial dispersion during low Reynolds number Taylor-Couette flow: intra-vortex mixing effects. Chemical Engineering Science 52, 3303-3310.

Chen, Y.M., Pearlstein, A.J., 1987. Viscosity-temperature correlation for Glycerol-Water solutions. Industrial \& Engineering Chemistry Research 26, 1670-1672.

Coeuret, F., Legrand, J., 1981. Mass transfer at the electrodes of concentric cylindrical reactors combining axial flow and rotation of the inner cylinder. Electrochimica Acta 26, 865-872.

Cohen, M., Maron, D.M., 1990. Analysis of a rotating annular reactor in the vortex flow regime. Chemical Engineering Science 46, 123-134.

Coles, D., 1965. Transition in circular Couette flow. Journal of Fluid Mechanics 21, 385-425.

Desmet, G., Verelst, H., Baron, G.V., 1996a. Local and global dispersion effects in Couette-Taylor flow-I. Description and modelling of the dispersion effects. Chemical Engineering Science 51, 1287-1298.

Desmet, G., Verelst, H., Baron, G.V., 1996b. Local and global dispersion effects in Couette-Taylor flow-II. Quantitative measurement and discussion of the reactor performance. Chemical Engineering Science 51, 1299-1309.

Desmet, G., Verelst, H., Baron, G.V., 1997. Transient and stationary axial dispersion in vortex array flows-I. Axial scan measurements and modelling of transient dispersion effects. Chemical Engineering Science 52, 2383-2401.

Drozdov, S.M., 2002. A numerical investigation of a modified Couette-Taylor apparatus with application to industrial mixing. Theoretical and Computational Fluid Dynamics 16, 17-28.

Enokida, Y., Nakata, K., Suzuki, A., 1989. Axial turbulent diffusion in fluid between rotating coaxial cylinders. American Institute of Chemical Engineers Journal 35, $1211-1214$

Forney, L.J., Pierson, J.A., 2003. Optimum photolysis in Taylor-Couette flow. American Institute of Chemical Engineers Journal 49, 727-733.

Forney, L.J., Skelland, A.H.P., Morris, J.F., Holl, R.A., 2002. Taylor vortex column: large shear for liquid-liquid extraction. Separation Science and Technology 37 2967-2986.

Giordano, R.C., Giordano, R.L.C., Prazeres, D.M.F., Cooney, C.L., 1998. Analysis of a Taylor-Poiseuille vortex flow reactor-I: flow patterns and mass transfer characteristic. Chemical Engineering Science 53, 3635-3652.

Giordano, R.C., Giordano, R.L.C., Prazeres, D.M.F., Cooney, C.L., 2000a. Analysis of a Taylor-Poiseuille vortex flow reactor-II: reactor modeling and performance assessment using glucose-fructose isomerization as test reaction. Chemical Engineering Science 55, 3611-3626.

Giordano, R.C., Giordano, R.L.C., Cooney, C.L., 2000b. Performance of a continuous Taylor-Couette-Poiseuille vortex flow enzymatic reactor with suspended particles. Process Biochemistry 35, 1093-1101.

Grohmann, A., Reiter, M., Wiesmann, U., 1981. New flocculation units with high energy efficiency. Water Science and Technology 13, 567-573.

Haim, D., Pismen, L.M., 1994. Performance of a photochemical reactor in the Taylor-Goertler vortical flow. Chemical Engineering Science 49, 1119-1129.

Haut, B., Amor, H.B., Coulon, L., Jacquet, A., Halloin, V., 2003. Hydrodynamics and mass transfer in a Couette-Taylor bioreactor for the culture of animal cells. Chemical Engineering Science 58, 777-784.

Hosokawa, Y., Furuta, H., Ishida, S., Kaneko, K., 1975. Diffusion phenomena in viscous solutions. Kanazawa Daigaku Kogakubu Kiyo 9, 161-167.

Iosilevskii, G., Brenner, H., Moore, C.M.V., Cooney, C.L., 1993. Mass transport and chemical reaction in Taylor-vortex flows with entrained catalytic particles: applications to a novel class of immobilized enzyme biochemical reactors. Philosophical Transactions: Physical Sciences and Engineering 345, 259-294.

Judat, B., Racina, A., Kind, M., 2004. Macro- and micromixing in a Taylor-Couette reactor and their influence on the precipitation of barium sulfate. Chemical Engineering \& Technology 27, 287-292.

Jung, W.M., Kang, S.H., Kim, W.S., Choi, C.K., 2000. Particle morphology of calcium carbonate precipitated by gas-liquid reaction in a Couette-Taylor reactor. Chemical Engineering Science 55, 733-747.

Kataoka, K., Takigawa, T., 1981. Intermixing over cell boundary between Taylor vortices. American Institute of Chemical Engineers Journal 27, 504-508.

Kataoka, K., Doi, H., Hongo, T., Futagawa, M., 1975. Ideal plug-flow properties of Taylor vortex flow. Journal of Chemical Engineering of Japan 8, 472-476.

Kataoka, K., Ohmura, N., Kouzu, M., Simamura, Y., Okubo, M., 1995. Emulsion polymerization of styrene in a continuous Taylor vortex flow reactor. Chemical Engineering Science 50, 1409-1416.

Lee, S., Lueptow, R.M., 2004. Rotating membrane filtration and rotating reverse osmosis. Journal of Chemical Engineering of Japan 37, 471-482.

Liu, C.I., Lee, D.J., 1999. Micromixing effects in a Couette flow reactor. Chemical Engineering Science 54, 2883-2888.

Lueptow, R.M., Docter, A., Min, K., 1992. Stability of axial flow in an annulus with a rotating inner cylinder. Physics of Fluids A 4, 2446-2455. 
Moore, C.M.V., Cooney, C.L., 1995. Axial dispersion in Taylor-Couette flow. American Institute of Chemical Engineers Journal 41, 723-727.

Moritz, H.U., Kossak, S., Langenbuch, J., Rink, H.P., Jung, W.A., 1999. Taylor reactor for reactions with viscosity changes. DE 19828742 A1, BASF Coatings AG.

Ohmura, N., Kataoka, K., Shibata, Y., Makino, T., 1997. Effective mass diffusion over cell boundaries in a Taylor-Couette flow system. Chemical Engineering Science 52, 1757-1765.

Ohmura, N., Noui-Mehidi, M.N., Sasaki, K., Kitajima, K., Kataoka, K., 2004. Mixing characteristic in a conical Taylor-Couette flow system at low Reynolds numbers. Journal of Chemical Engineering of Japan 37, 546-550.

Ohmura, N., Suemasu, T., Asamura, Y., 2005. Particle classification in Taylor vortex flow with an axial flow. Journal of Physics: Conference Series 14, 64-71.

Pudjiono, P.I., Tavare, N.S., Garside, J., Nigam, K.D.P., 1992. Residence time distribution from continuous Couette flow device. The Chemical Engineering Journal 48, $101-110$.

Racina, A., Kind, M., 2006. Specific power input and local micromixing times in turbulent Taylor-Couette flow. Experiments in Fluids 41, 513-522.

Racina, A., Poarnik, M., Kind, M., 2005. Experimental study of macro- and mesomixing in a Taylor-Couette reactor by laser-induced fluorescence (LIF). Chemie Ingenieur Technik 77, 1621-1626.

Recktenwald, A., Lücke, M., Müller, H.W., 1993. Taylor vortex formation in axial through-flow: linear and weakly nonlinear analysis. Physical Review E 48 4444-4453.
Rotz, C.A., Suh, N.P., 1976. New techniques for mixing viscous reacting liquids. Part I. Mechanical means to improved laminar mixing. Polymer Engineering and Science 16, 664-671.

Rotz, C.A., Suh, N.P., 1979. Vortex motions induced by V-grooved rotating cylinders and their effect on mixing performance. Transactions of the ASME 101, 186-192.

Schwille, A., Mitra, D., Lueptow, M., 2002. Design parameters for rotating cylindrical filtration. Journal of Membrane Science 204, 53-65.

Soos, M., Wu, H., Morbidelli, M., 2007. Taylor-Couette unit with a lobed inner cylinder cross section. American Institute of Chemical Engineers Journal 53, 1109-1120.

Syed, A., Früh, W.G., 2003. Modelling of mixing in a Taylor-Couette reactor with axial flow. Journal of Chemical Technology and Biotechnology 78, 227-235.

Tam, W.Y., Swinney, H.L., 1987. Mass transport in turbulent Couette-Taylor flow. Physical Review A 36, 1374-1381.

Wei, X., Takahashi, H., Sato, S., Nomura, M., 2000. Continuous emulsion polymerization of styrene in a single Couette-Taylor vortex flow reactor. Journal of Applied Polymer Science 80, 1931-1942.

Wiener, R.J., Snyder, G.L., Prange, M.P., Frediani, D., Diaz, P.R., 1997. Period-doubling cascade to chaotic phase dynamics in Taylor vortex flow with hourglass geometry. Physical Review E 55, 5489-5497.

Yim, S.S.S., Lo, M.Y.A., Titchener-Hooker, N., Ayazi-Shamlou, P., 1998. The dependence of residence time distribution on flow in co-axial cylinder device. Bioprocess Engineering 19, 221-227. 